\title{
Describing a COVID-19 Case and Presenting the Proposed Plan to Recovery
}

\author{
Ahmed Nadhmi Al-Doori ${ }^{1}$ and Dina S. Ahmed ${ }^{2, *}$ \\ ${ }^{1}$ Biomedical Engineering and Mechanics, College of Engineering, Virginia Tech University, \\ Blacksburg, VA 24061, United States \\ ${ }^{2}$ Department of Medical Instrumentation Engineering, Al-Mansour University College, Baghdad 64021, Iraq
}

\begin{tabular}{ll}
\hline \multicolumn{1}{c}{ Article's Information } & \multicolumn{1}{c}{ Abstract } \\
\hline Received: & The current outbreak of serious respiratory syndrome (aka COVID-19) has \\
18.03 .2021 & resulted from a novel coronavirus (SARS-CoV-2). This infectious disease was \\
Accepted: & classified as a pandemic by the World Health Organization (WHO) because it \\
22.04 .2021 & threatens public health and life worldwide. Recently, restrictions in many \\
Published: & countries are applied to detect the infected individuals, isolate them, and attempt \\
23.04 .2021 & to find appropriate treatments that can help decrease the disease's severe \\
Keywords: & symptoms. Regardless of the conducted efforts, the number of reported cases of \\
Coronavirus & coronavirus infections is still growing up. \\
COVID-19 & \\
Alternative & \\
Traditional & \\
Nutritional & \\
Herbal & \\
Medicine & \\
Treatment &
\end{tabular}

DOI: 10.22401/ANJS.00.5.04

*Corresponding author: dinasaadi86@gmail.com

\section{Introduction}

The analysis of the situation occurs here. They say understanding the problem is half the answer, and I believe that understanding the virus is a necessary prerequisite to comprehend the nature of the protocol discussed. COVID19 is a viral disease caused by SARS CoV-2, a virus that belongs to the coronaviruses family. The virus enters the body when it sticks to the mucous membranes of the nose, throat, or conjunctiva after traveling through contaminated air droplets. From this point on, the incubation period begins and lasts up to 14 days. Transmission is no longer a threat after the incubation period ends, which explains why patients are asked to quarantine for 14 days; however, symptoms can still persist. Those infected go through three stages. The first is the early infection phase, which is when the virus is still in the upper respiratory tract. Symptoms begin to appear in this stage, but for some individuals, COVID-19 never yields any symptoms. Typically, they start 2 to 14 days after infection, and can include fever, fatigue, joint pain, headache, dry cough, possibly diarrhea, and the loss of taste and smell. Approximately 7 days after symptoms start, if met with weak resistance from the immune system, the virus changes location to begin the second stage: the pulmonary phase. The virus moves to the lungs and results in a production of phlegm and pus from the dead cells fighting the infection in the alveoli. Dry cough turns to heavy wet cough and at the advanced level of this phase, results in difficulty breathing and mostly causes patients to be hospitalized. Those who deteriorate further develop Acute Respiratory Distress Syndrome (ARDS) and are known to be in the hyperinflammation phase. Loss of control over the infection can suddenly happen in this phase to result in multiple organ failures and death. It is true that this information sounds daunting, but it is worth mentioning that a very small percentage of infected patients reach the third phase, and half of them manage to recover, despite the difficulties associated [1].

What makes dealing with COVID-19 problematic is the lack of a direct cure. Although vaccines are becoming more and more available, viral infections generally remain a constant threat since there is no antiviral, unlike the case in bacterial infections where a doctor can prescribe an antibiotic accordingly [1]. This problem motivates the look for other methods that can aid in treatment, given that a virus like SARS-CoV-2 is constantly changing. I believe that a big contributor to the confusion seen in the world today is the absence of information regarding the treatment of COVID-19. Most of what is available from disease centers is about the prevention methods, which are extremely difficult to implement and maintain as proven by the surging cases. Therefore, I turned my focus on nutritional remedies, alternative medicine, and herbal 


\section{Al-Nahrain Journal of Science}

Special Issue: COVID-19, April 2021, pp. 17-20

remedies even if they are not endorsed or fully proven. The goal of this plan is to prevent the virus from reaching advanced pulmonary phase by boosting the immune system and limiting the viral infection. This paper's primary focus will be recovery and treatment, not prevention since it is widely discussed already.

\section{The Second Half: the Protocol}

The process to meet the challenging goal of the plan can be represented with a charming analogy. Assume that the virus is an invading enemy. The virus has attacked the empire of the body from an unguarded fort: the exposed mucous membranes. This enemy that is the virus, replicates itself in each city it controls, represented by the invasion of individual body cells and the emergence from them with higher numbers. In order for the body to defeat this slick invader, one can treat the situation like a defensive war from the perspective of the patient. To achieve victory as a patient, three requirements must be met:

- Intelligence: prior knowledge and ongoing awareness of the invaded territories as well as the enemy's condition and movement.

- Deep Defense: constant resistance and depletion of the enemy's supplies, equipment, or resources.

- Counter Offense: attacking the enemy's weakened points.

The body enters an alarmed state from the moment the infection begins, even if symptoms have not started to appear yet. Hence, the patient should also be focused and disciplined to help the body overcome the egregious task of eradicating the disease [2]. By implementing the three mentioned principles simultaneously, the patient will be in synch along the response within against COVID-19.

\section{Intelligence}

A pulse oximeter and a thermometer are necessary to monitor the condition continuously.

Ongoing information about the vital signs of the patient is an essential step towards knowing what action to take for treatment; medical instruments provide that knowledge, but some are not as accessible as others. For this review's protocol, oximeters and thermometers will be the instruments utilized since COVID-19 infects the lungs and as a result, the two most relevant variables are blood oxygen saturation level and body temperature. Thermometers are one of the most accessible medical instruments, yet oximeters are just as available but do not seem to have the same amount of recognition. Under this pandemic, oximetry can be very useful to monitor the blood's oxygen saturation $\left(\mathrm{SpO}_{2}\right)$ from home and avoid the development of the fatal silent hypoxemia [1]. Throughout the periodic checking, which should be done every few hours when normal or even more when unwell, the following considerations have to be in mind:
1. Regarding the oximeter: An oxygen saturation percentage or $\mathrm{SpO}_{2}$ above $95 \%$ is normal according to Yale Medicine $^{1}$ and Healthline ${ }^{2}$. On the contrary, the American Lung Association sets the normal level much less around $90 \%$. Because those with respiratory problems tend to have a lower normal $\mathrm{SpO}_{2}$, a value between $90 \%$ and $95 \%$ would qualify as acceptable for a COVID-19 patient. Lung exercises and deep breathing techniques can restore levels that are slightly below $95 \%$ back above it; as a matter of fact, the effect of deep breathing is almost instantaneous in this range. However, when there is a difficulty in completing a deep breath, and the $\mathrm{SpO}_{2}$ is low due to phlegm, clearing the lungs through coughing will be the way to boost $\mathrm{SpO}_{2}$ levels up. Doctors recommend contacting medical help when the $\mathrm{SpO}_{2}$ is below 90\%, but not everyone has access and there is a widespread shortage of beds ${ }^{3}$. When clearing the lungs, if there is difficulty in coughing, the patient can have someone else perform chest percussion to aid in the expulsion of phlegm. Chest percussion is done by tapping the back of the patient from the lower back up to the upper chest in order to shake the phlegm and aid its release. In the case where there is no one around to help, the patient can lay on the belly for a short time and the force of gravity will ease up the phlegm and make coughing easier [2]. It is urgent that one minimizes the intake of cough medications without expert consultation. Cough suppressants, on one hand, keep the phlegm trapped inside the lungs which causes more fluid buildup in the alveoli and deprives the body from oxygen; on the other, cough expectorants can lead to excessive coughing and undesirable side effects. It is advisable that one takes cough suppressants in the late time only to help sleep and rest, and take phlegm expectorants to help loosen mucous early in the day. The rule of thumb is to use a suppressant for dry cough and the nighttime, and an expectorant for wet cough and daytime [3].

2. Regarding the thermometer: The body temperature of a healthy individual at homeostasis is $37{ }^{\circ} \mathrm{C}(98.2 \mathrm{~F})$. When the individual is undergoing an infection, a rise in temperature occurs. If the fever is above $38^{\circ} \mathrm{C}(100.4 \mathrm{~F})$ but below $38.9{ }^{\circ} \mathrm{C}(102 \mathrm{~F})$, it is recommended to avoid rushing for fever and pain relievers [3]. The body raises its own temperature for a reason, to fight the infection, since higher temperatures weaken foreign pathogens, damage their enzymatic pathways, and boost the body's immune function. However, if the temperature reaches a point above $40{ }^{\circ} \mathrm{C}(104 \mathrm{~F})$, that's when fever relievers or wet blankets are preferable according to a study published by a team from the University of Miami. The referenced study provides strong evidence for letting fevers ride up to the point of 40 ${ }^{\circ} \mathrm{C}$, and shows that being strict about antipyretic treatment can be deadly in severe cases of infection. ${ }^{4}$ Attempting to return the body to a normal temperature early on when a viral infection is recent masks one of the best weapons 


\section{Al-Nahrain Journal of Science}

Special Issue: COVID-19, April 2021, pp. 17-20

against the infection, and provides a more favorable environment for viruses to thrive as a result.

\section{Deep Defense}

The body defense mechanisms are strengthened when certain practices (in nutrition and lifestyle) are abandoned, and others are implemented. Vitamin A, B, C, D, and E alongside the trace minerals are crucial for holding ground against the infection and are attained through Essential Nutrient Remedy.

Although there are currently no evidence from randomized controlled experiments to directly show a role for vitamins and minerals in the fight against COVID-19, there are strong scientific evidence, based on clinical studies of infection and ARDS, to indicate a role for vitamins and minerals supplementation, also known as immunonutrition, in turning the outcome of COVID infection to the better. Before going into detail about what to seek and what to turn away from nutrition-wise, there are necessary lifestyle changes that need to be mentioned. The most important tip to remember is to stay hydrated; water maintains homeostasis by controlling the fever, thinning the mucus for easier expulsion, and supporting the immune system functions. Besides that, rest is also necessary for prioritizing energy expenditure on fighting the infection [4].

\section{What to Avoid?}

- A sugar spike, the sudden rise of blood sugar, weakens the immune system and provides energy for the invading virus to reproduce, truthfully the worst possible combination of effects. Glucose metabolic pathways have key roles in initiating the immune response against viral infections, and their disruption often means an uncontrollable response that is often deadly [4]. When bad carbohydrates are consumed, which are carbohydrates that are digested quickly like sweets and beverages, the glucose metabolic pathways are disrupted to lead an uncontrollable immune response and a surge in signaling molecules known as cytokines. According to several studies, findings lead to the hypothesis that the main cause of death of COVID-19 is ARDS with cytokine storms [4]. Therefore, one must minimize sugar or even cut it completely. In addition, it is recommended to stay away from white rice and white bread especially when infected with the virus. In summary, one should avoid any form of carbohydrates that does not contain other nutrients like fiber and is not digested over a longer period of time. It is better to replace sugar with honey, juices with fruits and vegetables, white rice with brown rice or other whole grains, and white bread with whole wheat bread.

- Red meats, especially beef and ham, at normal conditions put their consumers at risk of heart attack and stroke. When the consumer is infected with COVID-19, the risk is much higher. At advanced stages of the disease, blood clots from the damaged lung tissues circulate the blood vessels; therefore, avoiding red meats to prevent a high cholesterol can mean the difference between life and death. Red meat should be replaced with fish, chicken, or turkey.

\section{What to Take on?}

- Various colorful vegetables and fruits like carrots, spinach, kale, cantaloup, and mangos contain the antioxidative beta-carotene, which transforms into vitamin $\mathrm{A}$ in the body. This fat-soluble vitamin helps repair damaged cells and functions as an antiinflammatory, meaning that it has roles in immune system modulation such as boosting the efficacy of antibodies. Although its direct link to COVID-19 is still being investigated, the resulting lung damage may in part be resolved through vitamin A-dependent mechanisms, and the differentiation of immune cells improved when its intake is sufficient. Because vitamin A is fat-soluble, consuming it with healthy fats such as olive oil can aid in its absorption significantly [4].

- The consumption of B vitamins is essential in the proper activation of immune functions, and the regulation of energy metabolism. In addition to participating in red blood cells production and the maintenance of the lymphatic system ${ }^{5}$, the B-complex is consequently pivotal in managing cytokine levels, as a result of its involvement in both immunity and metabolism. Cytokines are the proinflammatory signaling proteins that storm the body in late stages of the infection [4]. B vitamins are water soluble, and are found together in similar sources like seafood, poultry, legumes, and fortified cereals.

- Vitamin C is the go-to vitamin for those infected by the flu or common cold; so does it hold the same status when facing SARS-CoV-2? In the NCBI publication by Hoang et al, multiple studies are listed that display positive roles of high-does vitamin $\mathrm{C}$ application in critically ill patients of sepsis and viral respiratory infections. Vitamin $\mathrm{C}$ has powerful antioxidative properties, which eliminate free radicals and alleviate oxidative stress associated with tissue damage and other complications. Since COVID-19 typically leads to similar outcomes, vitamin $\mathrm{C}$ could be a valuable therapeutic approach in preventing them ${ }^{6}$. More clinical trials are underway to determine the direct link between vitamin $\mathrm{C}$ and COVID-19. All citrus, as well as green and red peppers, contain high amounts of that watersoluble nutrient [5].

- Many strong studies have suggested that vitamin D deficiency, a fat-soluble vitamin, is directly linked with severe cases of upper respiratory tract infections and COVID-19. Vitamin D has antimicrobial effects by preserving junctions between epithelial cells, which tend to be disturbed by pathogens like viruses, and by enhancing innate and adaptive immunity. It also modulates the pro-inflammatory response by decreasing the number of cytokine production, a process that can prevent the development of ARDS and other deadly 


\section{Al-Nahrain Journal of Science}

Special Issue: COVID-19, April 2021, pp. 17-20

COVID-19 related complications [6]. Vitamin D is manufactured in the body with sun exposure, or attained through dairy and eggs.

- The key to immune health is vitamin E. It regulates and enhances both cell-mediated and humoral immune functions. Vitamin $\mathrm{E}$ is found in almonds, walnuts, avocado, basil, and spinach.

\section{Counter Offense}

Certain herbal plants contain phytochemicals that target the virus to compromise its functions and decrease the severity of the symptoms. This section is known as Herbal and Spicing Remedy.

- Primarily, the early infection phase of the upper respiratory tract requires the intervention of compounds that control the replication of the coronavirus. Green tea contains epigallocatechin gallate (EGCG), a compound that inhibits virus proliferation, or replication. Green tea can be consumed in tea bags or as matcha powder.

- If the symptoms persist due to the continuous replication of the virus, licorice tea is considered as one of the most important respiratory health supporting remedies. It contains glycyrrhizin, which has been shown to inhibit viral adsorption and penetration. Also known as glycyrrhizic acid, this compound prevents viruses from invading body cells. Licorice tea is consumed as tea bags, or as a homemade extract from smashed licorice roots.

- Chamomile tea is typically consumed in the nighttime due to its calming effects. It has antioxidant and antiinflammatory properties that target cold related symptoms. The sedative effect makes it ideal for enhancing rest, and on top of that, it is used as a healing medicine.

- Garlic and onion can be used as the backup remedies when the virus moves to the pulmonary phase. They both act as mucus thinners, stimulate T-cells that fight the virus, and reduce stress hormones in the body to maintain normal functioning. Quercetin, a phytochemical found in both, is the main player behind the listed functions and also has various anti-inflammatory roles.

All the remedies mentioned were implemented in the daily meals of the family. Some were cooked as part of a recipe, spicy shrimp fajita for example, and others were taken directly such as an afternoon cup of licorice. Fortunately, these beneficial nutrients are found in a wide range of foods and can be morphed to fit the culture and preference of the patient. Of course, while keeping in mind to stay away from sugar and red meats. Through this program, my family ultimately healed in two weeks while enjoying newly cooked foods.

\section{Conclusions}

Since the finally-approved drug for this virus is still absent, and a sharp increase in infection numbers is taking place, it becomes urgent to present possible alternatives. The body defense mechanisms are strengthened when certain practices (in nutrition and lifestyle) are abandoned, and others are implemented. Despite that many of the listed treatments and remedies are not proven clinically for COVID-19, many of them have shown positive roles in similar situations. Additionally, given the global shortage of beds and the limited access to healthcare, the last thing to do would be to stay idle.

\section{References}

[1] Alsayed R.; Kadhom M.; Yousif E. and Sabir; "An Epidemiological Characteristic of the COVID-19 Among Children”, Lett. Appl. Nano Bio Science, 9(3), 1156-1164, 2020.

[2] Leila M. and Sorayya G.; "Genotype and phenotype of COVID-19: Their roles in pathogenesis", J. Microbiol Immunol Infect. 10, 2020.

[3] Hamming I.; Timens W.; Bulthuis M.; Lely A.; Navis G. and van Goor H.; "Tissue distribution of ACE2 protein, the functional receptor for SARS coronavirus. A first step in understanding SARS pathogenesis", J. Pathol. 203(2), 631-637, 2004.

[4] Alsayed R.; Ali A.; Makia R.; Kadhom M.; Raheem R.; Al-Obaidi O.; Hadi A.; Sabir D. and Yousif E.; "Challenges Facing Iraq to Tackle the Spread of COVID-19: An Overview", J. Uni Anbar Pure Sci. 14(2), 22- 27, 2020.

[5] Hadi A.; Kadhom M.; Hairunisa N.; Yousif E. and Mohammed S.; "A review on COVID-19: origin, spread, symptoms, treatment, and prevention", Biointerface Res. Appl. Chem. 10, 7234-7242, 2020

[6] Dijkman R.; Jebbink M.; Gaunt E.; Rossen J.; Templeton K.; et al.; "The dominance of human coronavirus OC43 and NL63 infections in infants", J Clin Virol. 53(2), 135-139, 2012.

[7] Hadi A.; Kadhom M.; Yousif E. and Hairunisa N.; "In COVID-19 time, how to protect myself and others? a review", Jurnal Biomedika dan Kesehatan. 3(3), 153$158,2020$. 